\title{
Experiences of Individuals Living with Chronic Mental Illness in Northern Namibia
}

\author{
Ndapeua Shifiona ${ }^{1, *}$, Marie Poggenpoel ${ }^{2}$, Chris Myburgh ${ }^{3}$ \\ ${ }^{1}$ School of Nursing, Faculty of Health Sciences, University of Namibia, Namibia \\ ${ }^{2}$ Department of Nursing, Faculty of Health Sciences, University of Johannesburg, South Africa \\ ${ }^{3}$ Department of Educational Psychology, Faculty of Education, University of Johannesburg, South Africa
}

Copyright@2019 by authors, all rights reserved. Authors agree that this article remains permanently open access under the terms of the Creative Commons Attribution License 4.0 International License

\begin{abstract}
The aim of this study was to explore and describe the lived experiences of individuals living with chronic mental illness, and the experience of those caring for them. A qualitative, explorative, descriptive, and contextual research design was used. Data was collected from four (4) multiple case studies which comprised of twenty-four (24) participants, utilizing in-depth phenomenological interviews with individuals living with chronic mental illness; one focus group interview with family members from each case, community members and health care workers. Interviews and focus group discussions were tape-recorded and transcribed verbatim. Data was analyzed by means of Tesch's open coding method. Three (3) main themes were identified; (a) experience of ineffective individual coping related to living with chronic mental illness, (b) experience of alteration in family processes related to the presence of long term mental illness, and (c) experience of individuals' impaired social interaction related to substance abuse and dysfunctional system evidenced by verbalized discomfort in social situations. The study concluded that an understanding of patients' experience by mental health nurses will enable them to facilitate the development of constructive interaction between individuals living with chronic mental illness and caregivers.
\end{abstract}

Keywords Chronic Mental Illness, Mental Health, Lived Experience

\section{Introduction}

Despite social changes and scientific advancements, mental illness continues to be one of the most personally devastating and costly type of illnesses in many countries. Findings from a study [20] on public conceptions about mental illness revealed that the illness is regarded as a very threatening, fearful challenge, and not an idea to be entertained lightly about anyone. Emotionally, it represents a loss of what people consider to be distinctively human qualities of rationality and free will, and there is a kind of horror and dehumanization.

Garrison [14] stated that mental disorders do not choose their victims; they occur in all cultures, at all ages and in both sexes. The sad thing is that there is too little attention being paid to helping people with mental illnesses. Among the African communities there is poor understanding about chronic mental illness which consequently results in ill treatment and discrimination of the patients by the family as well as community members. For example, a patient may be handcuffed to the ground and made fast for extended periods. Handcuffing and tying of individuals with mental illness to trees or heavy objects in order to immobilize them - especially those that are violent - is common in northern Namibia.

An illegal practice of incarcerating patients living with mental illness was recently uncovered in a local church. Four patients were found, padlocked with chains to tree trunks and other objects, after they sought spiritual healing from the pastor for mental - and other illnesses [16]. These malpractices can be attributed to a lack of understanding among family and community members of what mental illness is all about, as well as the varying views people have about mental illness in general.

Mental illness is highly stigmatized in some cultures and having a family member with a mental illness can affect one's social status and ability to marry. As a result, family members may consider it their responsibility to care for a mentally ill relative at home, only turning to a mental health agency when in dire need [15] One of the key parts in the process of subsuming mental disorders within socio cultural settings is the role of the family. The extent of family support for people living with psychiatric disabilities has often been regarded as a major factor in the rehabilitation process. Family support, which is easily available in developing countries, especially in rural settings, is the anchor for treatment and rehabilitation of 
individuals with mental illness in outpatient management [34].

Among the Oshiwambo speaking people in Namibia, some people strongly believe in bewitchment, especially after being struck by mental illness. Some individuals living with mental illness will first consult traditional healers to be reassured about who the cause of all the sufferings is, and for the "cursed object" to be removed from the body, before they report to the hospital. The "object" is believed to be foreign, and inserted into the sufferers' body through an invisible opening. The sufferer usually claims that the foreign object is the cause of their mental illness. Individuals living with chronic mental illness require highly structured, meaningful activities that must be provided by the family or a community day programme, which creates additional burdens for caregivers $[40,11]$ Such programmes do not exist in northern Namibia were the study was conducted.

The mental health situation in Namibia is said to be alarming, especially in the rural northern regions, where individuals living with chronic mental illness are severely neglected [37]. Being a mentally ill patient in Namibia often means a permanent fight against stigma and a daily struggle to make ends meets. These individuals speak of life with little social support from family members and friends, and no possibility of getting a paid job. They encounter ignorance even in professional health care surroundings. In the northern part of Namibia where the study was conducted, some people put more faith in traditional healers because they are accessible, even though many recognise the tremendous healing effects of western medicine. This is because traditional healers understand that for the Ovambos, illnesses are more than symptomatic disorders of the physical body and healing; more than the prescription of appropriate drugs. Even when the Ovambos use health care facilities regularly, some would continuously turn to an 'onganga' (traditional healer) in case of mental illness [8]

Within some Namibian communities, a culture that is open to offering support rather than shunning those who do not behave in predictable ways, is absent. In future, the thinking about community-based care needs to include representations from all parts of the community affected by caring for people with mental illnesses. The comprehensive understanding of a patient's mental illness as well as a thorough knowledge of the person's cultural context is valuable, as this affords better collaborations between the individual living with chronic mental illness and the health care workers.

Mental health service in Namibia is currently limited to two tertiary, mainly curative, institutions: Windhoek and Oshakati. The rest of the country is under-served. This, in turn, has an adverse impact on the involvement of the family and community in patient care. It also counteracts reintegration and rehabilitation of individuals living with chronic mental illness back into the community after discharge. Due to budgetary constraints mental health services in Namibia are low on the priority list. The services that are available are highly centralized, leaving the majority of the population without access to treatment. The few private psychiatrists and psychologists available can only cater for those able to afford their services. Thus Namibia remains short of skilled mental health care practitioners.

The provision of psychiatric services in Namibia, especially in the rural area, is inadequate. In the northern part of the country there is only one referral hospital with a mental health care unit, which is serving all the northern regions, with a total population of approximately 780149 [ 32] Individuals living with mental illnesses have to travel long distances to reach the health care facilities. The lack of financial resources, transport, and sometimes support from family and community members, can lead to defaults and exacerbation of the condition. There is a dire need for family and community inclusion in the provision of mental health care to individuals living with chronic mental illness. This should lead to services being better tailored to people's needs and better utilised. All the aforementioned factors underscore the importance of family and community involvement in the care of the mentally ill patient.

Sibbald [33] stated that in Namibia, mental health and physical health are very separate in the health care structure and there is tremendous stigma around mental illnesses and HIV in most places This notion is further supported by Haufiku [16] in his report on the "desperate need for mental health facilities" where he alludes that throughout Namibia there are no public, psychiatric health care facilities available at community level. Consequently, the effectiveness of community-based interventions aimed at increasing integration among people living with chronic mental illnesses, might be impeded by the experience of stigmatisation, and failing to address issues of stigmatisation may serve to reinforce the sense of marginalisation they experience [31].

Inadequate mental health services in the northern part of Namibia (previously referred to as Owamboland) signifies the importance of assessing individuals living with chronic mental illness, family and community members, as well as health care workers' views on living with chronic mental illness. Epidemiological data for specific mental illnesses are not available and while the World Health Organization (WHO) is committed to obtaining such data worldwide, this undertaking has yet to reach Namibia [13]. The results will justify the development of intervention strategies that will enable mental health nurses to promote, maintain and restore the individual living with chronic mental illness' mental health as an integral part of health and wholeness.

\section{Materials and Methods}

This was a qualitative study with an exploratory, 
descriptive, and contextual in focus as described by Chinn and Kramer [6]. It explored and described the lived experiences of individuals living with chronic mental illness in northern Namibia. In-depth, phenomenological interviews were conducted with four (4) discharged individuals living with chronic mental illness and their family members at their homes. Focus group interviews with community members and health care workers were conducted in order to elicit their experiences of living with and caring for someone living with chronic mental illness.

Observations were carried out in the form of field notes at participants' homes while focus group interviews with health care workers took place at the health care facility. The researcher carefully examined key concepts in order to find the appropriate characteristics and attributes as well as their relevance to the researched phenomenon, that is, the experience of living with chronic mental illness. The main objective was to explore participants' experiences of living with chronic mental illness and what they propose as a strategy to integrate individuals living with chronic mental illness into their community postdischarge from the hospital.

A purposive sampling was utilized to select the participants who were identified according to the following inclusion criteria: male or female individuals living with chronic mental illness between 18 - 60 years of age who have been discharged from the hospital; willingness to participate in the study; familiarity of the participant with the individual living with chronic mental illness; ability to speak Oshiwambo (local language) and/or English, and confirmed mental illness through medical diagnosis. Mason [22] stated that the logic of purposive sampling is that the researcher selects units, which will enable her to make meaningful comparisons in relation to the research question, the theory and the type of explanation one wish to develop.

The sample size was the results of data saturation because the emphasis was placed on quality rather than quantity. In this study the objective was not to maximise numbers but to become "saturated" with information on the topic namely, living with chronic mental illness [30]. For qualitative interviews, it is not the number of participants interviewed that is important, but rather the experience owned by the participant and the meaning attributed to it, that will be described as completely as possible. Four (4) multiple case studies comprised of twenty-four (24) participants in total were selected as a sample size (Figure 1).

This research study was conducted according to the basic ethical principles of research as outlined in the Belmont Report as well as the Declaration of Helsinki and Dhai and McQuoid-Mason [5, 9, 10, 43]. A consent form was designed and addressed to participant who showed the ability to read and follow explanations. For participants who were unable to read, verbal explanations were provided to them. Seeing that all potential research participants in the healthcare settings are vulnerable by the nature of their compromised health status, approval from the Ethical Clearance Committee of the Faculty of Health Sciences of the university was first obtained before commencement of this study.

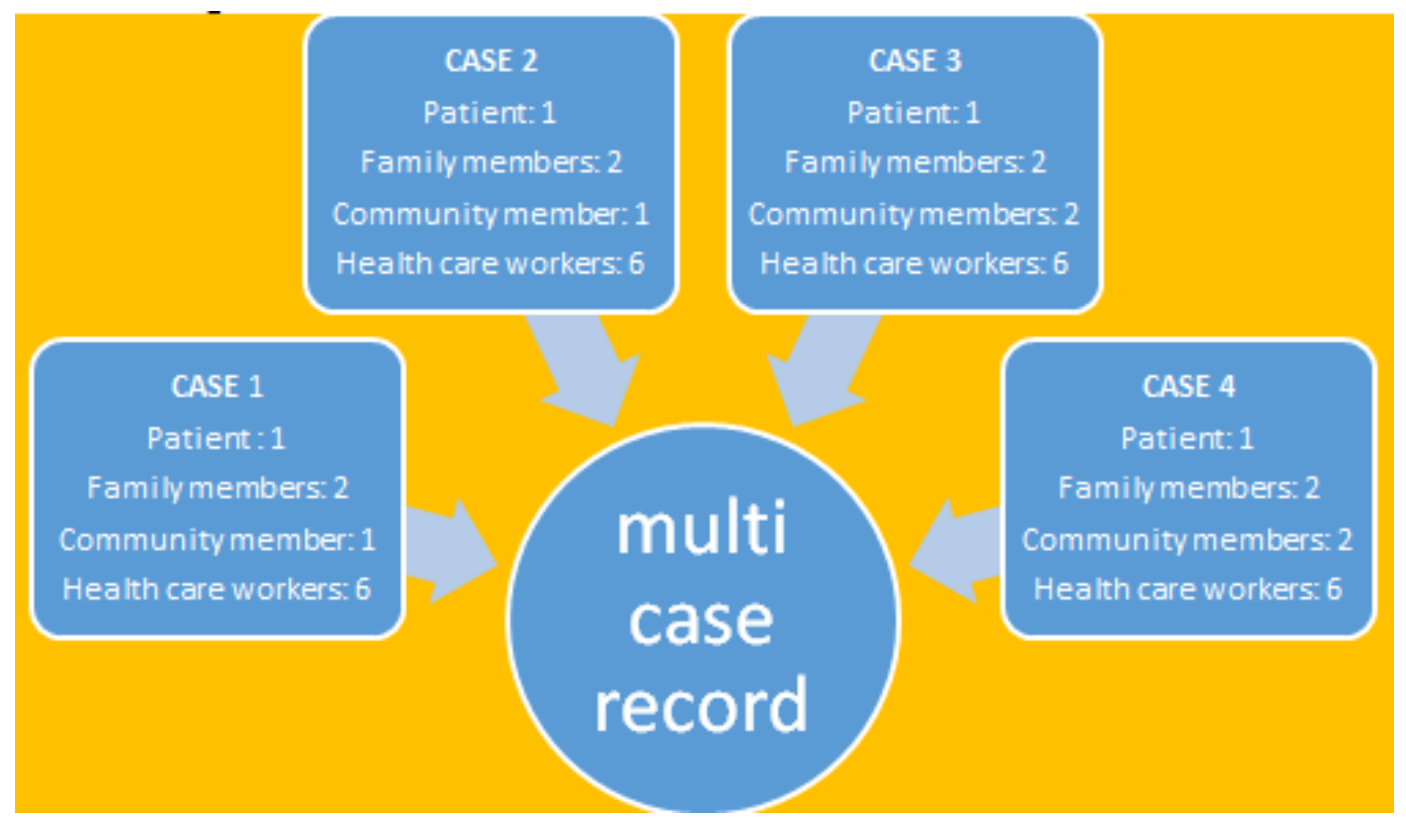

Figure 1. Schematic presentation of each multi-case record 
Written permission to conduct the research study was obtained from the Ministry of Health and Social Services, Namibia. The researcher also adhered to stipulations as outlined in the Ministry of Health and Social Services Research Management Policy as well as in the Guidelines on Clinical trials in Human Participants [27]. The research objective was articulated verbally and in writing so that they were clearly understood by the participants [7]. Written permission to proceed with the study as articulated was received from participants. Permission was also requested from all participants to audiotape the interviews. The right to anonymity and confidentiality was respected by de-linking participants' identity from the individual responses and by keeping the data as confidential as possible.

Data collection utilised the following multiple methods: in-depth phenomenological interview; focus group interviews; pilot study, field notes and observations. Two central questions were asked during the interview to explore the individuals' experiences about living with chronic mental illness namely, (i) "how is it for you to live with chronic mental illness?" and (ii) "how can you be assisted to become part of your family and community?"

The questions for family members were: (i) "how is it for you to have a relative living with chronic mental illness?" and (ii) "what do you think can be done to improve individual's living with chronic mental illness' day-to-day life situation?" Questions for community members were: (i) "how is it for you to have a community member who is living with chronic mental illness?" and (ii) "how can the community be assisted to accept individuals living with chronic mental illness and to improve their day-to-day life situation?”

For health care workers the following questions were posed; (i) "how is it for you to care for an individual living with chronic mental illness?” and (ii) "how can you be assisted to be able to help individuals living with chronic mental illness to integrate successfully into their community?"

Trustworthiness was ensured by applying the following four criteria as proposed by Lincoln and Guba and affirmed by van der Zouwen [9, 19, 38] in his model (Quality criteria for Naturalistic Inquiry) namely, truth value (credibility); applicability (transferability); consistency (dependability); and neutrality (confirmability). The activities that the interviewing researcher engaged in to attain credibility (truth value) of the findings and interpretations included; prolonged engagement with participants, triangulation by using multiple data collection methods, peer debriefing with study supervisors, member checking in order to clarify the interpretations of the participants' experience, keeping of a reflexive diary and avoid misinterpretations of the collected data. Applicability (transferability) was ensured by the provision of a dense descriptions of the participants' demographics and thick descriptions of the findings to enable other researchers interested in making a transfer, to reach conclusions about whether transfer in other contexts can be contemplated as a possibility. Consistency (dependability) was ensured through the provision of a complete descriptive methodology, literature control and verbatim quotes from case study interviews. Neutrality (confirmability) was ensured by the detailed discussion of the whole research process namely data collection, data analysis, discussion of the findings and recommendations.

Tesch's eight steps and Merriam's approach was used in combination as a method of data analysis [7, 26]. A protocol for data analysis was provided so as to guide the analysis process by the researcher as well as by the independent coder. The researcher and the independent coder held consensus discussions to compare their individually analysed data. The experiences of individuals living with chronic mental illness will be highlighted below.

\section{Results}

The main findings from interviews and focus group discussions are presented together, below. From the raw database, a set of three (3) themes and accompanying categories were identified. Themes identification process was conducted as follows: first each of the four case study was analyzed separately through reading and rereading of the raw data to get a sense of a whole. Each interview script was reviewed to evaluate and critically analyze what it is all about. The researcher focused on the underlying meaning in the story as presented by the participants during the interview or focus group discussions. After all the transcripts were reviewed, the researcher made a list of all the topics, clustering together similar topics, which again forms columns that were later arranged into major topics. A list of emerging topics was coded, organized and checked for emerging categories. The researcher than sought for categories that have internal convergence and also those with external divergence, meaning that, categories should display internal consistent but should also be distinctive from one another. The researcher proceeded to find the most descriptive wording for the topics and turned them into categories. Categories that related to each other were grouped together. Finally, all identified categories were reorganized and refined until final three (3) themes were formulated. The three themes for each individual case were first analyzed separately and across the four multiple cases. A protocol for data analysis was provided to guide the analysis process by the researcher as well as by an independent coder. The researcher and the independent coder held consensus discussion to compare their individually analyzed data.

On the basis of the three (3) themes, the voices of the participants across the four multiple cases were presented: 
1) Experience of ineffective individual coping related to living with chronic mental illness as reflected in an individual's inability to handle chronic mental illness and an inability to establish and maintain meaningful relationships;1.1) Experience of an individual's inability to establish and maintain meaningful relationship;

2) Experience of an alteration in family process related to the presence of long-term mental illness in a family member;

3) Experience of individuals' impaired social interaction related to substance abuse, and dysfunctional system evidenced by verbalizing discomfort in social situations. Highlights from each theme and related categories (where applicable) are discussed and supported with evidence from the literature.

Under the first theme, individuals living with chronic mental illness' daily lives were characterised by ineffective coping styles, which at times were accompanied by impaired interaction and negative behaviour patterns that foster specific life turmoil and difficulties of living. Learning to cope and live with chronic mental illness is stressful for most people. It leads to anxiety and unhealthy behaviours.

The following quotation is supporting the above notion:

"Deep in my heart it feels as if I am locked up in prison, I am trapped and disabled by this illness" [Participant 01]

A person is worried about who will care for him and how the illness will progress. What a person feel and think, and how one deals with those emotions, can affect the individual's ability to cope with a long term condition in many ways. An ineffective coping style leads to an unproductive manner of relating to life experiences. Each individual possesses a repertoire of coping skills that is used to relate to life experiences or to attain desired life conclusions.

\subsection{Participants Express the Inability to Handle Chronic Mental Illness}

Ineffective coping contributed to hardship living and drove the individual living with chronic mental illness into the execution of unacceptable activities such as burning of other people's properties.

"I've gone to an extent of destroying uncle's wife's kitchenware during the relapsing episodes and I am feeling bad about it. At times I get a feeling that Satan is the leading cause to all these inappropriate actions. Sometimes I realise that what I am about to say or do is wrong or unacceptable but I just wouldn't stop carrying it out" [Participant 2, 4]

Similarly, for family and community members, the caring task was also not easy. It demands an abundance of patience and tolerance from the rest of the family. The unavailability of human and material resources to adequately address the needs of the patients is complicating the day-to-day lives of these individuals, which is clearly illustrated in the following passage:

"There has been a marked increase in the number of in individuals living with chronic mental illness, with few health care workers running the unit. In these situations, controlling patient's behaviours as well as caring for them has become extremely difficult. The nature of the patients' uncontrollable behaviours prompts health care workers to put patients in isolation rooms to minimise and stop untoward behaviours. This is not caring at all!" [Focus G Group Discussion: health care workers]

\subsection{Individuals Living with Chronic Mental Illness Are Unable to Establish and Maintain Meaningful Relationships}

Patients experience problems related to relationships. These are due to the nature of the symptoms, like violent or aggressive behaviours, or an inability to play the expected role of husband or wife.

"No one would love to marry a mentally ill person". [Participant 3]

This phenomenon surfaced in almost all four cases. They are living a terrible, frightening, and lonely predicament Participants pointed out that due to the chronic mental illness, they experienced major losses, felt disconnected socially, marginalised economically and stigmatised because of living with chronic mental illness. One patient expresses it as follows;

"When I come back home, they make me angrier. When I am angry I leave the house. I can stay away for a week but when I come back, they take me to the hospital. It makes me very angry. This breaks up the relationship between me and members of my family because we do not communicate. I am angry and they too are afraid of me". [Participant 4]

For some patients, there is an inner struggle that keeps individuals living with chronic mental illness away from sharing with others; they feel no one will understand their plight. The following extracts fit the above category:

"I feel lonely and useless; it's very painful to think about what I am going through". [Participant 1, 3]

The family members may feel guilty about the illness and feel anguished for not knowing how to deal with the patient's behaviour.

"Acceptance of individuals living with chronic mental illness in the community remains a problem. When they, for instance, attempt to join a group of people, you will notice people whispering into each other's ears and making non-verbal gestures about him. Even those who know him will pretend not to know him". [Focus group: 


\section{Community Member]}

The widespread lack of empathy and understanding from members of the family, community members, and employers, is a direct result of not only discrimination but also ignorance about the plight of individuals living with chronic mental illness.

Patients as well as family members rarely seek or receive information, which makes them feel insecure concerning the best way to care for their relative Negative attitude towards patients is a common phenomenon as reflected in the passage below;

“... again lack of insight into the illness among community members causes division between a patient and his relatives. Some friends will for instance tell him that, hey brother, it is your sister, the nurse who is not good. He will believe them and you will always be the one at fault”. [Focus group: Community Member ]

Family members may constantly blame themselves for the progression of the illness. A family member could eventually feel threatened by the patient's untoward behaviors to such an extent that adequate support may not be fully rendered to the patient.

\subsection{Experience of an Alteration in Family Process Related to the Presence of Long-term Mental Illness in a Family Member}

Participants describe the negative impact of prolonged illness on their lifestyles, first as individual caretaker and secondly as a family unit. Sometimes family members have to take turns of caring for this individual by taking leave of absences from work to accompany him to the hospital for follow-ups; or attending him at home when he is unable to do so. Even when he is able, there is constant fear that he might relapse and harm himself or others as evidenced by the following remark.

"It is very difficult to have such a patient in your family" [Family member]

Another participant expresses it as follows:

"Due to misconceptions and lack of insight into this illness, some people believe that it is always mothers who carry the disease to their offspring and therefore the woman should carry the burden alone. This has led to conflicts in some families" [Family member]

Parents experience guilt and blame themselves for their child's illness, seeing it as a sign of genetic weakness or poor parenting. This is evidenced in one of the parents' remarks when she repeatedly put the blame for her child's illness on her own shoulders. She stated that:

"Sometimes I do think that perhaps I am the cause of his illness. I am blaming myself for this ..." [Family member]

One community member has this to say:

"It is a heavy burden to the affected family members because a person could be a productive member in that specific family but now, instead of the family benefitting from his service they are losing out economically as well as emotionally" [Focus Group: Community member].

Another participant described it as follows:

"Having someone with this condition is a heavy burden to the affected family. Emotionally, family members are also disturbed to such an extent that they may become less productive in their work performance. Coping with someone suffering from an illness of this nature is impossible if there is no support for the family. In this part of the country, from whom will you get the support?"[Family member]

One participant's description of the alterations read as follows:

"Because of this illness, my wife and my children are no more staying with me. They stay with my in-laws. Now and then I go and visit them or they will come and visit me. My mother does not like them at all. When my wife is around, she is always accusing them of stealing her things, or she is just suspicious of her. Every time when the telephone rings, she would come close to listen to what you are discussing. It is really frustrating. You are not free. I want to own a house but I cannot afford it, you see! You live like an intruder in your own mother's house. Is it not strange?"'Participant 01]

The patient felt threatened when he is in the company of family members.

\subsection{Experience of Individuals' Impaired Social Interaction}

Among individuals living with chronic mental illness, low self-esteem is a common phenomenon. Individuals with low self-esteem may fail to preserve or form new social relationships because they are less likely to seek out social activities. One participant describes the latter experiences as follows:

"Your movements are ever limited. When you come to social gatherings, many will stare at you as if they want to know why you are there" [Participant 01]

The poor self-concept associated with low self-esteem in patients living with chronic mental illness may hinder them from escaping the illness-focused world, making it difficult to immerse themselves in social interactions and maintain relationships as evidenced in the narratives from four participants

\subsection{Impaired Social Interaction by One Participant Resulted from Substance Abuse}

One of the participants describes his experiences with substances and the impairment it caused towards social interactions as follows: 
"You become confused, thinking that others are out to hurt you. While other people are trying to be in touch with you, you are distancing yourself from them. You are controlled by the substances. As people approach you, you become anxious, asking yourself, Mhmmm! What do they want to do with me?" [Participant 4]

Even though he temporarily enjoyed abusing substances with his peers, these behaviours have negative consequences for him as a person, physical as well as emotional. Under the influence of such substances, an impasse towards social interaction is created and this further leads to social isolation and impaired interaction.

One participant did not like the idea of being under the surveillance of his parents, and when he is on his own, he joins the wrong groups with whom he shares the dirty business of abusing substances. The latter statement is supported by the following remarks from one participant:

"When I come back home, they make me angrier. When I a am angry I leave the house. I can stay away for a week but when I come back, they take me to the hospital. It makes me very angry. This breaks up the relationship between me and members of my family because we do not communicate. I am angry and they too are afraid of me" [Participant 4].

One of the participants stated that the main motivation for using substances was to escape his own internal problems and to please his friends. He went on to state that when they angered him at home he will run away, spent about a week or two away and returns when he runs out of cash.

"I can stay away from home for weeks on end, but every time I come back home they will say I am mad. This angered me $m$ most to such an extent that I would run away from home back to the streets and continue smoking dagga trying to forget and it is also my way of coping with my problems" [Participant 4].

He did not have peace of mind at all and was hiding behind substances, which just worsens his situation. The person may also lie or engage in illegal activities to obtain drugs.

\subsection{Dysfunctional System Evidenced by Verbalizing Discomfort in Social Situations}

Individuals living with chronic mental illness find it difficult to socialise and interact with other people beyond their family circle. The stigma and discriminations attached to the illness has an enormous impact on the way other people perceive and/or interact with them. A perceived imaginary wall interferes with spontaneous interactions between the individual, family, community members and health care workers. The latter is supported by the following quote:

"It disturbs; it disturbs because you feel like you're not worth. You do not belong. You are not accepted as a human being. They discriminate against you and that's why it is very, very difficult for me to live with the illness. Even small children are using those bad names against me. They are told 'it is a mad person, ten minus ..., has lost control of his mind. And the children also, when I walk past they are just uttering the same statements and words. There is no respect, no matter how old you are, what age you are of which category your age group is, whether you are married or not, they do not care...." [Participant 1]

These expressions of helplessness and hopelessness are common among individuals living with chronic mental illness. They are also subjected to ill-treatment from health care workers; the group that is supposed to be on the frontline of fighting for their rights. The illnesses have forced many mentally ill persons to live in isolation and were not able to participate in social activities. One participant shared the following sentiments;

"A person will be walking around day - and - night, no rest; the illness is forcing her to walk around, even at places that are not known to them. You will feel for them; you know ..." [Participant 3].

When individuals living with chronic mental illness and their family members, for example, receive poor support from health care workers and from social networks, their coping strategies tend to stagger. A number of care-giving family members have experienced symptoms such sleeplessness, excessive irritability and inner unrest, which is an indication of their difficulties to cope. This has a negative impact on the quality of care and support they ought to render to the patient

These individuals express their need to be assisted in their search for mental health. Family and community members indicated the obstacles encountered when caring for someone living with chronic mental illness and has therefore strongly recommended that an intervention strategy is needed.

\section{Discussion}

Participants talked about fear of living with chronic mental illness, anger and sometimes feelings of guilt about their inability to cope with the disease pattern. Family members spoke about their inability to assist those living with the illness. This is what is called 'subjective burden' in the literature [28,29]. Coping is the most difficult part of the journey that most individuals living with chronic mental illness are enduring. The latter has been confirmed by Bradshaw, Roseborough and Armour [2] that these individuals "find themselves extremely overwhelmed by the disability when trying to understand what is happening, confused at times, disconnected from self and others and experiencing little sense of self efficacy". People living with chronic mental illness also develop disabilities that affect all spheres of their lives. 
This is due to a lack of acquisition of skills, or loss of skills due to disuse. Due to long-term illness, these individuals may stop taking care for themselves. They may become disinterested in social interactions and relationships, or be socially inept. During the period of hospitalisation, only few are exposed to life skills activities through the process of rehabilitation. The majority will spend their days idly. Health care workers describe the facilities they are working in as a trigger to fresh psychotic symptoms. A patient will be admitted in a somewhat manageable, but it will become worse by what the patient and fellow patients, are exposed to.

Becoming ill took them away from other people and resulted in disconnection. After a diagnose and living with the illness for a long time resulted in an isolated way of life, an unintended disconnection from other people accompanied by an inability to interact or to secure lasting relations with others. Bradshaw, Roseborough and Armour [2] in their study expresses similar sentiments that were verbalised by the participants namely, "dropping out of school” (an example in this study was participant 4 who dropped from a tertiary institution), "loosing job" for example participant 3 and 4, and "loosing friends" (participant 1 and 4); leading to boredom and a sense of alienation. These are deep emotional feelings that upset the individual living with chronic mental illness and lead to the development of feelings like unworthiness and hopelessness. The nature of the illness makes it extremely difficult to establish and maintain mutual relationship with others. For the patient, there are times when the need to form such a relation is there, but this is unfortunately preceded by the fear that he might not be understood, or no one would want to have a relationship with a mentally ill person. These types of feelings may be regarded as setbacks in the patient's efforts to establish and sustain meaningful relationships. The dark experiences of mental illness isolate the sufferer from the warmth of human affection [3]. Loosing friends was not only evident in individuals living with chronic mental illness alone, but also in family and community members as well. Family members and friends can become confused, frustrated, angry, and scared when they are not able to interact with the sufferer. The situation becomes even worse when they are confronted with bizarre behaviours that can erupt during an episode, making it hard for them to remain close.

Feelings of alienation, pain and ineffective coping were experienced across the board. Stobbe [35] shared similar sentiments that two-thirds of people affected by mental illness often feel lonely, or experience loneliness most, if not all of the time. From all the participants in the study, stigmatisation was found to be the root to problematic interpersonal relationships. It deprives individuals living with chronic mental illness their full measure of human dignity and participation in wider society. These individuals need to have a strong support system to feed their interest or engagement in life, which will sustain their morale and keep them going during the periods of adversity. But this does not happen.

WHO [41] concluded that disturbances to an individual's mental well-being can adversely compromise a person's capabilities to form and maintain meaningful relationships, leading not only to diminished functioning at the individual level, but also broader welfare losses on the household and societal level. Furthermore, the illness has a devastating impact on the patient's ability to carry out activities of daily living, self-care as well as participation in social life of which eventually contributes to the on-going level of disability. Estevam, Marcon, Antonio, Munari and Waidman[12] reported the concerns expressed by family members about the lack of knowledge concerning the meaning of mental illness. They felt despair when noticing the patient's change of behaviour, sorrow, anxiety, and many other feelings

In this study, due to family member's need to see recovery taking place, family members will take the mentally ill person to the healers, sometimes against what their religion proscribes; a desperate attempt on their side. At times traditional healers claim to have healing powers against mental illness and lure the affected family member/s into their practice. But sometimes, instead of addressing the illness as such, healing is said to take place only if the traditional healer is allowed to have sexual intercourse with the sufferer (if female) during the days or months of the so-called 'healing sessions'. Upon returning home, the illness remains unchanged but the person (if female) will take home another burden, like 'unwanted pregnancy'. Such incidents hurt family members and tear them further apart, blaming the person who initiated the service. They experience powerlessness and fear, which often results from families' realisation that they are dealing with a long-term recurrent illness [36].

The prolonged illness also leads to negative attitudes among some family members by perceiving the individual living with chronic mental illness as useless with no value on earth. Opinions from them are not validated, as is mostly evidenced by the negative remarks made against the individuals living with chronic mental illness. For many people, being hospitalised for a mental illness connotes the end of a productive lifestyle and the beginning of long term care. The patient may feel alienated from society. The family is disrupted when a member is hospitalised, and the longer the hospital stay, the more likely the family is to reassign the other member's roles and exclude the suffering person. The family's lifestyle and leisure activities are altered.

Mental illnesses are also said to be predictors as well as consequences of substance abuse [25]. One of the participants used it to escape from difficulties experienced at home with his parents. Chronic mental illness is closely related to the abuse of, and dependence on, both alcohol and other illicit drugs as a temporary hub away from enduring trouble. Constant parent-child conflict was 
common in one case. The parent-child conflict is associated with impulsivity, rebelliousness, and internalising behaviours, which are attributed to vulnerable personality common among delinquent and drug-using peers [18]. Addiction co-opts the brain's neuronal circuits necessary for insight, reward, motivation, and social behaviours. This functional overlap results in addicted individuals making poor choices despite awareness of the negative consequences [39]. On substance abuse, Stuart and Laraia [36) attribute this kind of behaviour to being an expression of rebelliousness against parental control and also a way of obtaining gratification. The drug becomes the centre of the user's life, despite adverse consequences [23]. Individuals living with drug addiction suffer, since the addicted persons are preoccupied with getting and maintaining their addiction at the expense of their family and friends. The addicted person becomes emotionally unavailable and not interested in previous relationships.

Patients as well as family members rarely seek or receive information from health care professionals about the meaning as well as the management of mental illness, of which makes them feel insecure concerning the best way to care for their relative. Angermeyer, Beck, Dietrich and Holtzinger [1] reveals that most patients living with mental illness expect negative reactions from public and concrete stigmatization. A family's dreams and plans for the future may be given up. Social roles are disrupted because often there is not enough money, or energy to devote to the sick person. Overall, stress from negative judgements, stigmatisations and rejections by friends and neighbours can negatively affect the health and functioning of family members.

All four (4) multiple cases portray a picture of patients, relatives, community members struggling to come to terms with chronic mental illness. These sufferings are not unique to Namibia alone. Caregivers experience a maelstrom of emotions as they struggle to understand what has happened to their loved ones. It therefore requires a high level of vigilance from all caretakers involved.

\section{Conclusions}

Individuals suffering from chronic mental illness as well as family members have trouble in their efforts to cope with mental illness. The widespread lack of empathy and understanding from family, community and health care workers is a direct result of not only discrimination but also ignorance about the plight of individuals living with chronic mental illness. The kind of support patients receive from family, community members, health care workers and from social networks on a daily basis seems inadequate. Hence, their coping strategies tend to stagger. Participants from all four multiple case studies acknowledged the importance of recognising an individual with long term mental illness as a unique individual with personal needs to be met., and who is constantly searching for acceptance within the family circle as well as within the community. Family and community involvement is therefore a fundamental ingredient in the management of chronic mental illness. High-level community mental health nursing interventions need to be initiated, supported and maintained in order to facilitate a better understanding into mental illness. By advocating positive interpersonal interaction between individuals living with chronic mental illness, their family members, community members and health care workers, the promotion of mental health will be facilitated.

\section{Acknowledgements}

We are very grateful to all for the valuable input and contributions to the success of this study.

\section{REFERENCES}

[1] M.C. Angermeyer, M Beck, S. Dietrich, S \& A. Holzinger, The stigma of mental illness: patient' anticipations and experiences. International Journal of Social Psychiatry, 50(2):153-162. Available from: http://www.ncbi.nlm.nih.g ov/. 2004.

[2] W. Bradshow, D. Roseborough, \& M.P. Armour, Recovery from severe mental Illness: The Lived Experience of the Initial Phase of the Treatment. International Journal of Psychosocial Rehabilitation, 1(10):123-131. 2006.

[3] E. Brohan, \& G. Thornicroft, Stigma and discrimination of mental health problems: workplace implications. Occupational Medicine, 60(6):414-420. Available from: http://www.occmed.oxfordjthenal.org/. 2010.

[4] M. Brune, M. Abdel-Hamid, C. Sonntag, C. Lehmkamper, \& R. Langdon. Linking social cognition with social interaction: non-verbal expressivity, social competence and "mentalising" in patient with schizophrenia spectrum disorders. Behavioural and Brain Functions, 5:6. Available from: http://www.behaviouralandbrainfunctions.com/ 2009.

[5] Bryn Mawr College. Ethics of Fieldwork. The Principles of the Belmont Report. Available from: http://www.brynmaw r.edu/ 2012.

[6] P. L Chinn, \& M.K Kramer. Integrated Theory and Knowledge Development in Nursing. $8^{\text {th }}$ edition. Atlanta: Elsevier. 2011.

[7] J. W. Creswell. Research design: Qualitative, Quantitative, and Mixed Method Approaches. Thousand Oaks: SAGE. 2009.

[8] G. Davies. The Medical Culture of the Ovambo of Southern Angola and Northern Namibia. University of 
Kent

Kantebury. Available from: http://lucy.ukc.ac.uk/csacpub/ Davies_thesis. 1994.

[9] B. DeLanda, The Belmont Report. History, Principles and Application. Research Compliance Office. Available from: http://www.humansubject.stanford.edu/ 2009.

[10] A. Dhai \& D. McQuoid-Mason. Bio-Ethics, Human Rights and Health Law. Cape Town: Juta. 2011.

[11] M. Eklund, Work status, daily activities and quality of life among people with severe mental illness. Qual Life Res (18):163-170. Available from: http://www.lup.lub.lu.se/ 2009.

[12] M.C. Estevam, S, S. Marcon, M.M Antonio, D.B. Munari, \& M.A.P. Waidman. Living with mental disorders: family members' perspective have on primary care. Rev Esc Enferm Usp. 45(3):677-684. Available from: http://www.scielo.br/ 2011.

[13] A. Feinstein. Psychiatry in post-apartheid Namibia: a troubled legacy. Psychiatric Bulletin. British Journal of Psychiatrists. Available from http://www.pb.rcpsych.org//ggi/content/full/26/8/310-a. 2002.

[14] P.J. Garrison. Making Mental Health a global priority: Why consumer, family member, citizen advocacy and action make a difference in service and policy development. World Mental Health Day, WHO .2008.

[15] H. Gravitz. The binds that tie-and heal: How families cope with mental illness. AVU Lib. Gateway, 34(2):70-76. 2001.

[16] M. Haufiku, M. Church unchains mental detainees. New Era Newspaper, Available from: http://www.newera.com. na/. 2012.

[17] M. Janik. Mental Health Week. New Era. Available from: http://www.newera.com.na/. 2008.

[18] E Lacourse, D.S. Nagin, F. Vitaro, S. Côte, L. Arseneault, \& R.E Tremblay. Prediction of early-onset deviant peer group affiliation: a 12-year longitudinal study. Arch Gen Psychiatry, 63:562-568. 2006.

[19] Y.S Lincoln. \& E. G. Guba, Naturalistic Inquiry. London: SAGE. 1985.

[20] B.G. Link, J.C. Phelan, A. Stueve, \& B.A. Pescosolido. Public Conception of Mental Illness in 1950 and 1966: What is mental illness and what is to be feared? Journal of Health and Social Behavior. 41(2):188-207. Available from: https://campus.fsu.edu/ 2000

[21] C. Marshall \& G.B. Rossman. Designing Qualitative Research. London: SAGE Publications. 2006.

[22] J. Mason. Qualitative Researching. Thousand Oaks, CA: Sage Publications. 2002.

[23] T. Mauro. The Many Victims of Substance Abuse. Psychiatry (Edgmont), 4(9): 43-51. 2007.

[24] T.V. McCann, D.I. Lubman, \& E. Clark. First-Time Primary Caregivers' Experience of Caring for Young Adults with First-Episode Psychosis. Schizophr Bulletin, March 2011; 37(2):381-388. 2009.
[25] J. R. Measelle, E. Stice, \& J.M. Hogansen. Developmental trajectories of co-occurring depressive, eating, antisocial, and substance abuse problems in female adolescents. Journal of Abnormal Psychology, 115:524-538. 2006.

[26] S. B. Merriam. Qualitative Case Study Research: a guide to design and Implementation. San Fransisco: Jossey-Bass. 2009.

[27] Ministry of Health and Social Services. Research Management Policy and Ministry of Health guidelines on clinical trials. Windhoek: MoHSS. 2003.

[28] W.K. Mohr, W.K. Johson's Psychiatric-Mental Health Nursing. Philadelphia: Lippincot Williams \& Wilkins. 2003.

[29] C. Olwit. Chronic Sorrow: Lived experiences of caregivers of schizophrenic patients in Butabika Mental Hospital, Kampala, Uganda. A dissertation submitted in (partial) fulfillment of the requirements for the Degree of Master of Nursing (Mental Health) of Muhimbili University of Health and Allied Sciences. Muhimbili University of Health and Allied Sciences. Available from:http://www.ir.muhas.ac.tz :8080/ 2012.

[30] D.K. Padgett. Qualitative Methods in Social Work Research. Los Angeles: Sage Publications.2008.

[31] P.N. Prince \& C.R. Prince. Perceived stigma and Community Integration among clients of assertive community treatment. Psychiatric Rehabilitation Journal, 25 (4):323-331. Available from: http://www.ncbi.nlm.nib. gov/ 2002.

[32] Regions of Namibia. Census Summary Results. Available from: http://www.npc.gov.na/census/2005.

[33] B. Sibbald. Building Namibia's HIV and mental health services. Canadian Medical Association Journal, 175(5):463. Available from: http://www.cmaj.ca/ 2012.

[34] V. Stanhope. Culture, Control and Family Involvement: A Comparison of Psychosocial Rehabilitation in India and the United States. Psychosocial Rehabilitation Journal. African University Library Gateway. 2002.

[35] E. Stobbe, Social Isolation and Mental Illness. Available from: http://www.brainblogger.com/ 2006.

[36] G. Stuart, \& M. Laraia, Principles and Practice of Psychiatric Nursing. St Louis: Elsevier Mosby. 2005.

[37] The Namibian Newspaper. Mental illness alarmingly underestimated in Namibia. Staff reporter. Available from: http://www.namibian.com.na/ 2012

[38] T. Van der Zouwen. Highlights of Naturalistic Inquiry, Doing Naturalistic Inquiry: (David, Edward Harris, Barbara Skipper \& Steve Allen, 1993). Available from: http://www.learninghistories.net 2004.

[39] N.D.Volkow, R.D. Baler, \& R.Z. Goldstein Addiction: Pulling at the Neural Threads of Social Behaviours. Neuron, 69(4):599-602. Volkow, N.D.; Baler, R.D. \& Goldstein, R.Z. (2011). Addiction: Pulling at the Neural Threads of Social Behaviours. Neuron, 69(4):599-602. 2011. 
[40] H.R. Winefeld, \& P.L. Burnett. Barriers to an alliance between family and professional care-givers in chronic schizophrenia. Journal of Mental Health, 5(3):223. 1996

[41] World Health Organization. Risks to Mental Health: An overview of vulnerabilities and risk factors. Background Paper by WHO Secretariat for the Development of a Comprehensive. 2012.

[42] Mental Health Action Plan. World Health Organization. Geneva, Switzerland. Available from: http://www.who.int/

[43] World Medical Association Declaration of Helsinki. Ethical Principles for Medical Research Involving Human Subjects. Available from: http://www.wma.net/e/policy: 15. 2008. 